\title{
Sterilisation in the laboratory autoclave using direct air displacement by steam
}

\author{
P. H. EVERALL, C. A. MORRIS, AND R. YARNELL \\ From the Public Health Laboratory and Department of Microbiology, \\ Shrewsbury and Copthorne Hospital (North), Shrewsbury, UK
}

SUMMARY A device using a steam injection funnel is described by means of which air can be $\stackrel{\dot{\omega}}{\longrightarrow}$ driven quickly and surely from an autoclave load. It is simple and inexpensive, necessitates no ? changes in the working routine of a microbiology laboratory, and does not interfere with the $\vec{P}$ operation of the autoclave in its normal mode.

While it is accepted that air must be removed from the chamber of a laboratory autoclave and from the interstices of its load if sterilisation is to be attained, the means of achieving this with certainty are usually not so well understood. For a variety of reasons the method of air removal so effective in a porous load autoclave is not suitable, and reliance is usually placed on downward displacement of the heavier air by the lighter steam. When the load consists of material for discard, contained in, say, a stainless steel bucket, sterilisation will depend on the production of sufficient turbulence in the steam in the chamber to dispel the air from the bucket. Without such turbulence it is hard to see why the heavier air should ever be displaced, so that much thought has been given to maximising turbulence by varying the position of the chamber steam inlet and the shape and size of the baffle plate; by removing the baffle plate (Scruton, M.W., personal communication 1975); by careful selection of the discard containers; and even by providing them with holes permitting the more easy escape of the air (Gillespie and Gibbons, 1975). Even so, with some containers, and some loads, air removal does not take place, and sterilisation is not achieved.

This paper describes a simple device in which the removal of air is accomplished directly by the incoming steam.

\section{Material}

AUTOCLAVE

A Dent and Hellyer steam heated autoclave was

Received for vublication 18 July 1977 used with internal dimensions $26 \times 26 \times 26$ in $\frac{\mathbb{D}}{2}$ $(0.7 \times 0.7 \times 0.7 \mathrm{~m})$. In normal use steam is admitted to the chamber through an entry port in the rear ${ }^{2}$ wall and dispersed by a baffle plate size 23.5 in $(600 \mathrm{~mm})$ square.

The autoclave was modified by inserting a ' $T$ " piece in the steam pipe just before it entered the chamber. From this ' $T$ ' piece an additional steam pipe complete with a hand-operated valve (v) was taken into the chamber, utilising for this purpose the $\mathbb{D}$ thermocouple entry port. Inside the chamber this second supply was terminated by fitting four Hansen ${ }^{1}$ 을 valves; the pipework to these and the valves themselves were kept as close to the chamber wall as possible. At the lowest point of this pipework was fitted a brass radiator air vent so adjusted as to be용 just sufficient to allow the discharge of condensate. A feature of the Hansen valve is that it is only when union is made between the male and female halves $\delta$ that steam can flow through it. The arrangement of 3 the pipework and valves is shown in Figures 1 and 2. 을

STEAM INJECTION FUNNELS

This name has been applied by us to the only part of the device which has had to be specially con- $\Omega$ structed. The funnels are 14 in overall in length so $N$ that when placed in the laboratory's discard buckets $N$ the stem protrudes some 2 in above the top of the $\sigma$ bucket. The stem has an outside diameter of 0.5 in

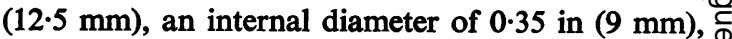
and a length of 10.5 in $(265 \mathrm{~mm})$. The bowl of the

${ }^{1}$ Made by the Hansen Manufacturing Company, Cleveland, Ohio and supplied by J. Leach and Company, Statham Street, Hanley, Stoke-on-Trent, UK. 


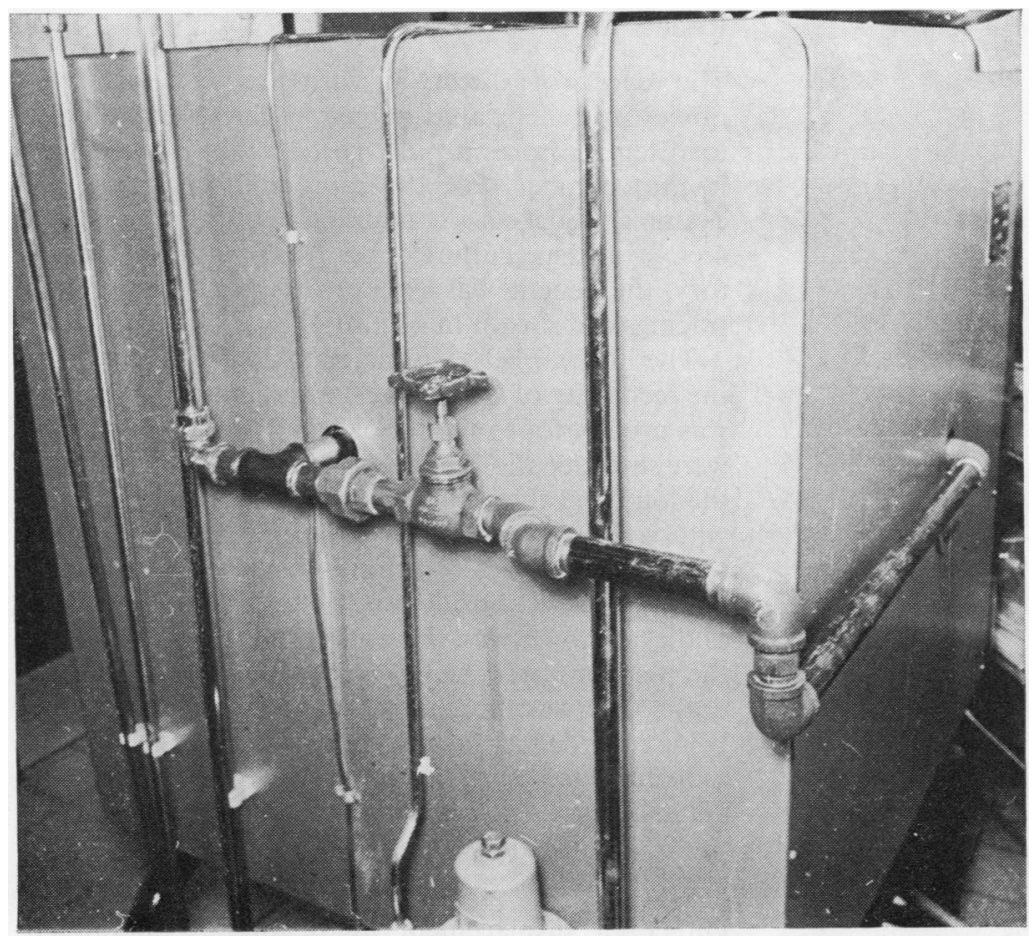

Fig. 1 Dent and Hellyer Protector autoclave showing modifications to pipework at rear and side.

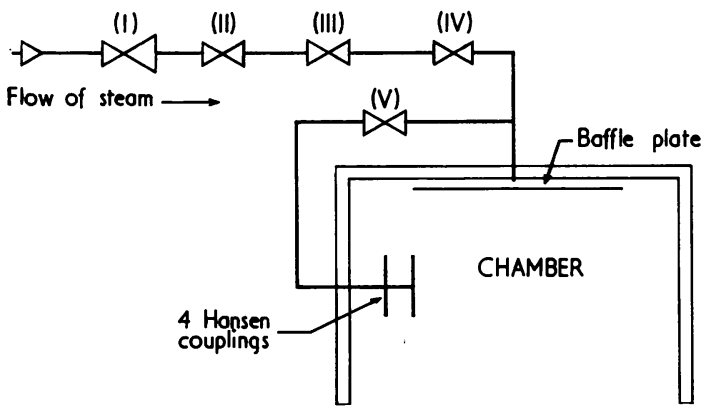

Fig. 2 Diagram of layout of pipework. $V=$ handwheel valve controlling steam supply to Hansen valves; I, II, III, and IV are, in order, a reducing valve, a solenoid operated valve, a manually operated valve on the fascia of the autoclave, and a reducing valve controlling the chamber pressure.

funnel is constructed from 16 gauge stainless steel in the form of a cone having a base diameter of 7.5 in $(190 \mathrm{~mm})$ and a vertical height of 3.5 in $(90 \mathrm{~mm})$. Twelve 3-mm holes are drilled in the wall of the funnel, spaced equidistantly from each other, and about 2 in $(50 \mathrm{~mm})$ from the base of the funnel bowl (Fig. 3).

\section{CONNECTORS}

Silicone rubber tubing is used to make connection between the Hansen valves and the 'funnels'. Each piece of tubing $(0.5$ in $(12.5 \mathrm{~mm})$ internal diameter) needs to be about 30 in $(740 \mathrm{~mm})$ in length. To one end is attached the female half of the Hansen valve.

\section{THERMOCOUPLES}

Temperature measurements in the loads were made using a Chessel 301 instrument using copper/constantan thermocouples: as the thermocouple entry port was not available for use these were inserted between the door seal and the chamber wall.

\section{Method of use}

IN THE LABORATORIES

Instead of providing simple buckets for specimens and other materials requiring sterilisation, these are supplied with a steam injection funnel in position. When full they are sent to the sterilising room where they are autoclaved. From there they are passed to the wash-up department where, after cleansing, the buckets with funnels in place are returned to he laboratories for re-use. 


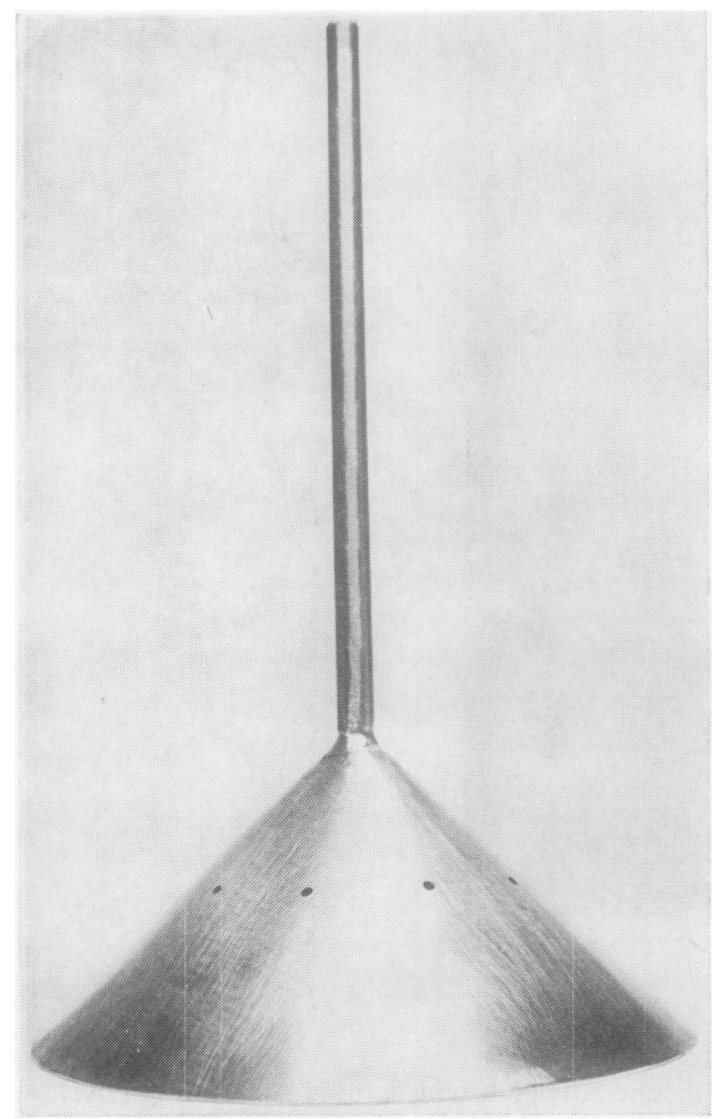

Fig. 3 Steam injection funnel.

IN THE STERILISING ROOM

A strip of autoclave tape is placed in position across the top of each bucket and the buckets are loaded into the autoclave. The newly fitted handwheel valve (v) is checked to ensure that it is closed. Union is made between the female half of the Hansen valve and the male half, and the other end of the silicone rubber tube is attached to the $50 \mathrm{~mm}$ of funnel stem protruding from each bucket. Up to four such buckets can be accommodated. The autoclave cycle is started up normally. Once the chamber drain thermometer registers $100^{\circ} \mathrm{C}$ the hand wheel valve (v) is opened. Steam then passes down the funnels, forcibly displacing the residual air from the load.

Temperature measurements were made from the chamber drain, the chamber space, and at various points in the load. For measuring load temperatures the thermocouple junction was placed inside one of the items making up the load. Coincidentally temperature measurements were made from similar containers and similar loads not equipped with the device.

\section{Results}

The effect of direct air displacement was very $\underset{\vec{*}}{\vec{*}}$ obviously beneficial as judged by the speed at which load temperatures rapidly rose to that of the steam in the chamber. For the more difficult loads, for example buckets containing rubber stoppered $4 \times \frac{1}{2}$ in tissue culture tubes from the virus laboratory, the benefit was greatest. Typical temperature tracings are shown in Figure 4.

The interval between the opening of valve (v) and the recording of 'sterilising' temperatures in the load was usually four minutes or less. Such temperatures were recorded more or less simultaneously from both the load and the chamber drain although otherwise, without the direct air displacement device, load ? temperatures lagged behind chamber drain temperatures for a considerable time. Where at the start of the holding period there was a discrepancy between the temperatures being recorded from load and drain this was because a higher temperature was being recorded in the load than in the chamber drain, a feature never normally seen in an unmodified autoclave at this stage in the cycle.

\section{Discussion}

Direct air displacement by steam has undoubted value in displacing air quickly and with certainty from a load of infected materials requiring sterilisation. The modifications necessary are not expensive and the use of steam injection funnels does not make it necessary to introduce unwelcome changes in the day-to-day routine of a microbiology laboratory.

Since the auxiliary steam supply to the funnels is 'teed off' from the supply pipe to the chamber it is subject to the same safety provisions as is the normal steam inlet (for example, it is not possible to get steam from the Hansen valves until the autoclave door is closed). Furthermore, if valve (v) is not open (or if the male and female halves of the Hansen valves are not connected) the autoclave functions in its normal mode.

Our recommendation that valve $(v)$ is not opened until the chamber drain reads $100^{\circ} \mathrm{C}$ is based on two considerations. The first is the necessity to lay down $\sigma$ a standard procedure easily followed by the auto- $N$ clave operator, and the second is based on the belief that a theoretical advantage should attach to filling the autoclave with steam before forcibly driving the air from the infected materials being sterilised.

In the sterilisation of plastic petri dishes by this method we expected difficulties resulting from the low melting point of the polystyrene of which they are made. However, the $3 \mathrm{~mm}$ holes in the funnels did not become blocked, the mass of polystyrene was easily removed at the end of the process, and those 

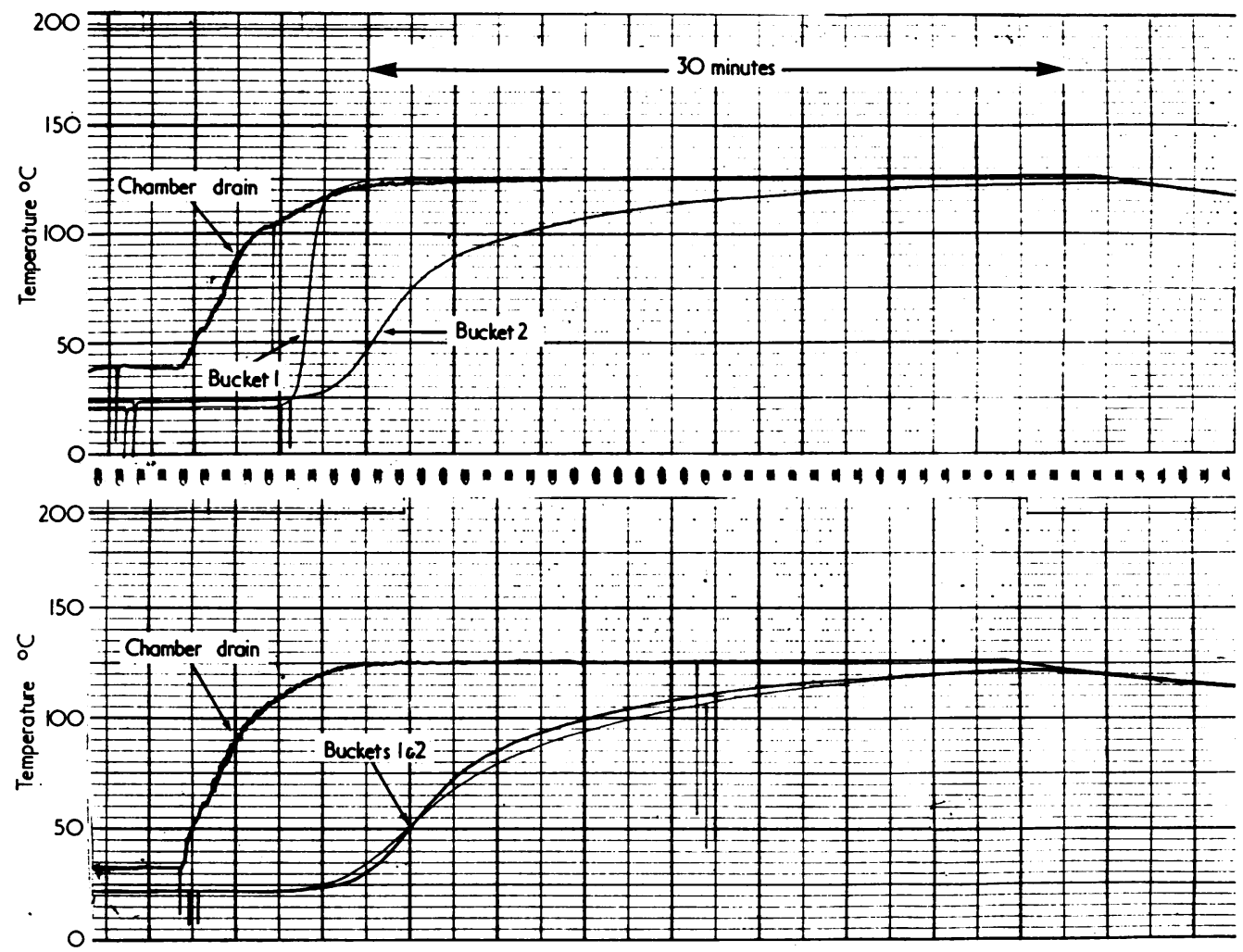

Fig. 4 Thermocouple tracings from two separate experiments using in both an identical load of two buckets equipped with 'funnels' and containing screwcapped $\frac{1}{4}$ oz bottles. Under normal operation (lower tracing) the temperature in neither bucket reaches that of the steam, whereas when the direct air displacement device is operated in bucket 1 (upper tracing) the temperature of its contents rises in 2 minutes to that of the chamber drain.

responsible for the cleaning of the buckets and funnels found the procedure completely acceptable.

The silicone rubber tubing used for connecting the Hansen valves to the funnels has proved excellent and has already, in this laboratory, withstood more than 300 autoclavings without any apparent deterioration, apart from a change in colour.

The manner in which we accomplished our purpose of introducing direct air displacement into this autoclave is not, of course, the best method of doing so but was a compromise between our desire to test such a system and at the same time maintain the autoclave in regular routine use without any major alterations being necessary.

Attention has been drawn in the past to the lack of adequate instrumentation on many laboratory autoclaves and to the operator being forced to rely almost solely on his previous experience in the judging of cooling times (Everall and Morris, 1975). Equally, most laboratory autoclaves at present in use tell the operator little or nothing of the temperatures in the load which he is attempting to sterilise; although some loads, because of their shape and nature (for example, tissue culture tubes), may present particular problems of heat penetration of which he is quite unaware. The use of direct air displacement funnels has the advantage that, because the air is forcibly driven out, the differences in steam penetration times into various loads are largely removed and this allows the operator to be confident that the loads are held at the sterilising temperature during the holding period.

\section{References}

Everall, P. H., and Morris, C. A. (1975). Some observations on cooling in laboratory autoclaves. Journal of Clinical Pathology, 28, 664-669.

Gillespie, E. H., and Gibbons, S. A. (1975). Autoclaves and their dangers and safety in laboratories. Journal of Hygiene, 75, 475-487. 